\title{
Bedingungsfaktoren der Fraktionsgeschlossenheit. Eine vergleichende Analyse der deutschen Länderparlamente
}

\author{
Christian Stecker
}

\section{Determinants of Party Unity. A Comparative Analysis of the German State Parliaments} Abstract: Using 2184 roll call votes (RCV) from 42 electoral terms this paper tests the influence of different factors on party unity in the German Landtage. It finds evidence that the strategic calculus behind RCV-requests is a crucial predictor for unity scores. The requesting party group is on average significantly more united than its parliamentary competitors. It also shows that government status, thin governmental majorities and a growing ideological distance between government and opposition increase unity. Electoral incentives, however, do not seem to influence voting behavior in the German Länder.

Keywords: Party Unity, Party Discipline, Roll Call Votes

Schlüsselwörter: Fraktionsgeschlossenheit, Fraktionsdisziplin, namentliche Abstimmungen

\section{Einführung}

Die hinreichende Geschlossenheit von Parlamentsfraktionen ist eine zentrale Voraussetzung dafür, dass das Repräsentationsideal des „responsible party government" funktioniert. Nur wenn Parteien durch einiges Handeln klar identifizierbar sind, können Wähler wirksam politische Aufträge erteilen und Verantwortung eindeutig zurechnen. Insbesondere die Regierung benötigt die Unterstützung ihrer Abgeordneten, um ihr Gesetzgebungsprogramm umzusetzen (Cheibub 2007, S. 132). Auch für die Fähigkeit von Parlamenten politische Expertise zu entwickeln (Patzelt 1998, S. 327; Schüttemeyer 1998) und stabile Entscheidungen zu fällen (Carey 2006), ist die Einigkeit der Fraktionen unabdingbare Voraussetzung.

Entsprechend gehört die Analyse des Abstimmungsverhaltens als Teil des Repräsentationshandelns von Parteien und Abgeordneten (vgl. Manow u. Zittel 2008) zu den klassischen Themen der Politikwissenschaft (siehe zu einem ausführlichen Forschungsüberblick Fritzsche 2009; Saalfeld 2005). Zwei weitgehend komplementäre Ansätze stehen dabei im Vordergrund. Soziologisch informierte Studien konzentrieren sich auf Normen und Sozialisationsprozesse, die das Verhalten im Parlament beeinflussen (z. B. Jensen et al. 2000). Rationalistisch motivierte Arbeiten (z. B. Carey 2007; Sieberer 2006b) beleuchten, welche institutio-

\footnotetext{
Für hilfreiche Forschungsassistenz danke ich Johannes Kuhn, Michael Schulze, Christin Skiera und Heiko Wenzel. Eine frühere Version des Aufsatzes wurde im AB-B Kolloquium des Mannheimer Zentrums für Europäische Sozialforschung vorgestellt. Ich danke den Teilnehmern sowie Alexander Busch, Sebastian Eppner, Erik Fritzsche, Johannes Freudenreich, Katja Heeß, Bernhard Miller, Monika Mühlböck, Jochen Müller und zwei anonymen Gutachtern für wertvolle Anmerkungen und Kommentare. Verbleibende Fehler gehen auf meine Kappe.
} 
nellen Anreize zur Geschlossenheit bestehen und wie sich Abgeordnete mit dem rationalen Teil ihres Verhaltens daran anpassen (teils synthetisierend: Saalfeld 1995b; Patzelt 2006; Kam 2009; Andeweg u. Thomassen 2011).

Die vorliegende Studie trägt zum rationalistischen Verständnis von Fraktionsgeschlossenheit in parlamentarischen Demokratien bei. ${ }^{1}$ Anhand von 2184 namentlichen Abstimmungen aus insgesamt 42 Wahlperioden aller 16 deutschen Länderparlamente wird untersucht, wie verschiedene Faktoren auf System-, Parlaments-, Partei- und Abstimmungsebene die Geschlossenheit einer Fraktion beeinflussen. Damit nimmt diese Studie eine x-zentrierte Kausalperspektive ein (Ganghof 2005, S. 80), das heißt, anstatt eine vollständige Erklärung des Phänomens anzustreben, soll der Effekt einiger weniger Einflussgrößen möglichst sicher abgeschätzt werden. Entsprechend liegt der Fokus auf der sorgfältigen Diskussion und Überprüfung dieser Faktoren. Von den Eigenheiten der konkreten Fälle muss dabei soweit wie möglich abstrahiert werden. Im Sinne einer kollektiven Forschungsstrategie ergänzt der Beitrag die bestehenden stärker fallstudienartigen Einblicke in die Fraktionsgeschlossenheit auf Länderebene (Davidson-Schmich 2006; Könen 2009) um eine variablenorientierte, quantitative Perspektive.

In vier wesentlichen Punkten geht der vorliegende Aufsatz über ähnlich motivierte Studien hinaus. Erstens erreicht er mit der Untersuchung der Landtage eine größere Kontrolle externer Störgrößen als bestehende international vergleichende Länderstudien. Dieser methodische Mehrwert ist insbesondere vor dem Hintergrund relevant, dass Geschlossenheitsforschung besonders auf gute Vergleichsbedingungen angewiesen ist. Der Vielzahl möglicher intervenierender Variablen auf Ebene des politischen Systems und des Parlaments steht häufig eine minimale Varianz der abhängigen Variablen gegenüber (siehe Sieberer 2006b, S. 161). Für Cheibub (2007, S. 134) ist die Heterogenität der Fälle sogar Grund genug, Erkenntnisse über institutionelle Effekte auf Geschlossenheit aus internationalen Untersuchungsdesigns vollständig anzuzweifeln.

Zweitens nutzt diese Untersuchung die Geschlossenheit einer Fraktion bei einer Abstimmung als Analyseeinheit. Dies erlaubt es, zusätzlich zur System- und Parteiebene Erklärungsfaktoren auf Abstimmungsebene einzubeziehen und lässt ein im Vergleich zu Aggregatdatenanalysen detaillierteres Bild entstehen.

Damit verbunden ist ein dritter Punkt. Dieser Aufsatz berücksichtigt zentrale Einwände gegen die Aussagekraft namentlicher Abstimmungen. Die Forschung zum europäischen Parlament hat jüngst darauf hingewiesen, dass das strategische Kalkül von Fraktionen beim Beantragen namentlicher Abstimmungen die Datenbasis entlang zentraler Hypothesen systematisch verzerren kann (Carrubba et al. 2006; siehe auch Hug 2010). Die vorliegende Studie kontrolliert dieses Risiko, indem sie zu jeder namentlichen Abstimmung zusätzliche Informationen über die beantragende Fraktion einbezieht.

$\mathrm{Zu}$ guter Letzt füllt dieser Beitrag eine empirische Lücke. Das Wissen über Fraktionsgeschlossenheit in den Länderparlamenten beruht bisher auf der Analy-

1 Neuere Analysen der Fraktionsgeschlossenheit im Vergleich von präsidentiellen und parlamentarischen Demokratien finden sich bei Kailitz (2008) und Carey (2007). Zu präsidentiellen Demokratien siehe Morgenstern (2004). 
se weniger Landtage (Könen 2009) oder eines nur geringen Anteils namentlicher Abstimmungen (Davidson-Schmich 2006). Hier können erstmals Daten zur Geschlossenheit auf Basis einer Vollerhebung aller namentlichen Abstimmungen aus allen 16 Landtagen über insgesamt 42 Wahlperioden zwischen 1990 und 2010 hinweg präsentiert werden.

Es zeigt sich, dass insbesondere die Fraktion von höherer Geschlossenheit profitiert, die die entsprechende namentliche Abstimmung beantragt hat. Auch Regierungsstatus, knappe Mehrheitsverhältnisse und eine wachsende ideologische Distanz zwischen Regierung und Opposition erhöhen die Geschlossenheit. Anreize des Wahlsystems lassen sich dagegen nicht am Abstimmungsverhalten ablesen.

Der Artikel ist wie folgt gegliedert. Im nächsten Abschnitt wird ein gängiges Modell zur Erklärung von Fraktionsgeschlossenheit vorgestellt. Anschließend werden darin eingebettete Faktoren diskutiert und Hypothesen formuliert, die an den Länderparlamenten überprüft werden können. Ausführlich wird darauf eingegangen, wie Fraktionsstrategien bei der Beantragung namentlicher Abstimmungen auf das Datensample und die Geschlossenheitswerte wirken. Der folgende Analyseteil präsentiert deskriptive Informationen sowie einen multivariaten statistischen Test der Hypothesen. Im Schlussteil werden die Ergebnisse zusammengefasst und diskutiert.

\section{Ein Modell zur rationalistischen Erklärung innerfraktioneller Geschlossenheit}

Innerfraktionelle Geschlossenheit ist ein mehrdimensionales Phänomen. So weisen Beiträge in Zeitungen und Talk-Shows, Anfragen und Redebeiträge im Parlament auf die Einigkeit einer Fraktion hin (vgl. Kam 2009). Wie Abgeordnete über kollektiv verbindliche Entscheidungen im Parlament abstimmen, gilt jedoch als wichtigste Dimension und steht in den meisten Untersuchungen, so auch hier, im Vordergrund.

Bei der Erklärung von Geschlossenheit hat Hazan (2006) in Anlehnung an Ozbudun (1970) die Unterscheidung zwischen den Faktoren Kohäsion (cohesion) und Disziplin (discipline) etabliert. ${ }^{2}$ Dabei ist Kohäsion, verstanden als die Ähnlichkeit politischer Präferenzen der Abgeordneten einer Fraktion, eine Bedingung ihres Abstimmungsverhaltens. ${ }^{3}$ Wären die politischen Präferenzen aller Fraktionsmitglieder identisch, würde sich Einigkeit quasi von selbst einstellen. Fraktionsdisziplin dagegen „starts where cohesion falters“ (Hazan 2006, S. 3). Stimmen

2 Zusätzlich kann Geschlossenheit auch durch (negative) Agendakontrolle gesichert werden, indem eine formale Abstimmung über intern umstrittene Gegenstände geschäftsordnungsrechtlich verhindert wird (Cox u. McCubbins 2005). Diese Form nimmt in der US-amerikanischen Parlamentsforschung einen prominenten Platz ein, spielt in Deutschland institutionell bedingt jedoch keine Rolle (Sieberer 2006a).

3 Für Hazan trennen beide Konzepte auch gleichzeitig die zwei grundlegenden Ansätze zur Erklärung von Fraktionsgeschlossenheit (Hazan 2006, S. 3-5). Kohäsion bezeichne neben „shared preferences" auch eine ähnliche Sozialisierung und geteilte Normen der Abgeordneten, die vorrangig im vorparlamentarischen Raum wirken. Die rationalistische Erklärung auf Basis institutioneller Anreize und individueller Handlungskalküle sei dagegen im Begriff der Disziplin zusammengefasst. Meines Erachtens treibt Hazan die sinnvolle analytische Unterscheidung dabei etwas zu weit. Rationalistische Erklärungen von Geschlossenheit fußen selbstverständlich sowohl auf Kohäsion - eng verstanden als Ähnlichkeit politischer Präferenzen - als auch auf Disziplin. 
Abgeordnete trotz divergierender Ansichten zu einem Gegenstand geschlossen ab, beruht dies auf positiven institutionellen Anreizen und negativen Sanktionen seitens der Fraktionsführung. ${ }^{4}$ Wie bereits in dieser Unterscheidung anklingt, werden Abstimmungen aus der Perspektive des rationalistischen Erklärungsmodells häufig als ein Spiel zwischen individuellem Abgeordneten und seiner Fraktionsführung (bzw. zwischen, frontbench" und ,backbench') gedacht, dessen aggregiertes Ergebnis am Niveau der Fraktionsgeschlossenheit ablesbar ist.

Dabei wird das individuelle Abstimmungskalkül zunächst dadurch beeinflusst, dass Abgeordnete bestimmte Ziele erreichen wollen - insbesondere wiedernominiert und -gewählt zu werden, öffentliche Ämter zu besetzen und politische Entscheidungen zu beeinflussen (vgl. Müller u. Strøm 1999). Der institutionelle Kontext bestimmt, wie sie diese Zieltrias aus vote, office und policy am besten erreichen können. Insbesondere bestimmt er, wie stark Abgeordnete dabei zu parteidienlichem Verhalten tendieren bzw. durch die Fraktionsführung dazu motiviert werden können. Die Fraktionsführung gilt als Vertreter des kollektiven Interesses der gesamten Partei. Sie versucht, ein einiges Auftreten zu gewährleisten, da davon Reputation und Wahlerfolg der gesamten Partei abhängen (Dittberner 2003; Eilfort 2003). Dergestalt kann Geschlossenheit als Kollektivgut begriffen werden, von dem alle Fraktionsmitglieder profitieren. Gleichwohl bestehen für einzelne Abgeordnete immer wieder Anreize, nicht zur Produktion dieses Gutes beizutragen, beispielsweise, wenn eine politische Maßnahme in ihrem Wahlkreis unpopulär ist. In der Summe kann dieses vereinzelte Trittbrettfahren dazu führen, dass zu wenig Geschlossenheit ,hergestellt' wird, worunter alle Fraktionsmitglieder zu leiden hätten. Dieses Risiko kann die Fraktionsspitze als „legislativer Leviathan " (Cox u. McCubbins 1993) desto einfacher minimieren, je mehr positive Anreize und Sanktionen ihr an die Hand gegeben sind. Im Idealfall können einzelne Abgeordnete ihre Ziele nur erreichen, wenn sie sich dem kollektiven Ziel unterordnen, als Partei einig aufzutreten (Patzelt 2006, S. 105).

Die Kombination aus ,carrots and sticks' kulminiert in der Möglichkeit die Vertrauensfrage mit einer Sachfrage zu verknüpfen (Döring u. Hönnige 2006; siehe für die deutschen Länder Reutter 2005). Dadurch verbindet die Regierung ihr Überleben mit der Verabschiedung einer Gesetzesvorlage. Mit einem Votum gegen die eigene Regierung würde ein Abgeordneter seine in Zukunft umsetzbaren politischen Ziele (Diermeier u. Feddersen 1998, S. 618) und seine im Rahmen der Regierungsteilhabe besetzten Ämter zur Disposition stellen. Durch eine eventuell folgende Wahl stünde zudem sein Mandat, wenn nicht gar die Fortsetzung seiner politischen Karriere auf dem Spiel (kritisch: Cheibub u. Limongi 2002). Im parlamentarischen Alltag dienen beispielsweise der Zugang zu Redezeit, Wahlkreisbesuche von Spitzenpolitkern oder Regierungs- und Parlamentsämter als An-

4 Es ist ein zentrales Problem der Geschlossenheitsforschung, dass dieser Unterscheidung empirisch kaum Rechnung getragen werden kann. Aus der bloßen Beobachtung des Abstimmungsverhaltens einer Fraktion können zunächst nur Aussagen über das Ausmaß der Geschlossenheit getroffen werden. Der Analyse bleibt jedoch verborgen, wie dieses Verhalten aus den Präferenzen einzelner Mitglieder und den ihnen gesetzten institutionellen Anreizen resultiert (Krehbiel 2000; Saalfeld 2005, S. 44-49). 
reize für loyale Fraktionsmitglieder (für weitere Möglichkeiten: Müller et al. 2001, S. 196).

Schwieriger wird die Sicherung von Geschlossenheit, wenn neben der Fraktionsführung weitere, konkurrierende Einflüsse auf das Abstimmungskalkül der Abgeordneten wirken. Diese bei John Carey (2007) als „competing principals“ figurierenden Akteure finden sich beispielsweise in machtvollen Interessenverbänden oder in (lokalen) Wählergruppen. Sie besitzen ihrerseits Ressourcen wie Wahlkampfmittel oder schlicht Stimmengewicht, mit denen sie um die Loyalität eines Abgeordneten werben können. Kommt es zum Konflikt zwischen den Präferenzen der Fraktionsführung und denen eines competing principals, muss ein Abgeordneter genau abwägen, welchen Prinzipal er zufrieden stellt. Hängt davon sein Mandat ab, wird er auch bereit sein, sich gegen die eigene Fraktion zu stellen.

Der institutionelle Kontext beeinflusst demzufolge in beträchtlichem Maße den Ausgang dieses gedachten Spiels zwischen Fraktionsführung und individuellen Abgeordneten. Im Folgenden diskutiere ich zentrale Variablen aus diesem Kontext, deren Einfluss anhand der deutschen Länderparlamente untersucht werden kann.

\section{Institutionelle Bedingungsfaktoren der Fraktionsgeschlossenheit}

Zentrale Voraussetzung für eine politische Karriere in parlamentarischen Demokratien ist ein Parlamentsmandat. Wer den Zugang zum Parlamentsmandat kontrolliert, kann das spätere Verhalten von Abgeordneten nachhaltig beeinflussen. Folgerichtig gelten Kandidatennominierung und Wahlsystem als besonders wichtige Faktoren für das Abstimmungsverhalten. Bestimmt die Fraktionsführung die Nominierung von Kandidaten, verfügt sie über ein sehr machtvolles Instrument, um Geschlossenheit zu erzeugen. Einerseits kann sie ex ante eine Vorauswahl politisch ähnlich überzeugter Kandidaten treffen (Bowler et al. 1999, S. 6) und andererseits ex post den Entzug der Nominierung als Drohung gegen Abweichler im Parlament ins Feld führen (Gallagher u. Marsh 1988, S. 15).

Der Einfluss des Wahlsystems auf das Repräsentationshandeln von Abgeordneten ist von Cain, Ferejohn und Fiorina (1987, S. 9) prägnant im Begriff der „personal vote" konzeptionalisiert worden. Das prominente Kontinuum zwischen parteizentrierten und kandidatenzentrierten Wahlsystemen (Carey u. Shugart 1995; Mitchell 2000; Norris 2004) bildet einen ähnlichen Grundgedanken ab. Demnach begünstigen kandidatenzentrierte Wahlsysteme (z. B. offene Listen) direkte Bindungen zwischen Abgeordneten und einzelnen (lokalen) Wählergruppen. Wem sie ihre Stimme geben, entscheiden Wähler hier nicht nur auf Basis der Parteizugehörigkeit, sondern auch anhand der Herkunft eines Kandidaten (Shugart et al. 2005), seiner wahrgenommenen Kompetenz (Mackenrodt 2008) oder seines Engagements für den Wahlkreis (Klingemann u. Wessels 2003). Entsprechend kultivieren Kandidaten diese parteiunabhängige Unterstützerbasis, was sich in erhöhter Wahlkreisarbeit und einer lokal orientierten Gesetzgebung niederschlagen kann. Konfligieren die Fraktions- und Wahlkreisinteressen bei einer Abstimmung, ist auch ein Bruch der Fraktionsgeschlossenheit durch diese Kandidaten möglich. 
In parteizentrierten Wahlsystemen verdrängt die Zugehörigkeit zur Partei dagegen individuelle Merkmale von Abgeordneten als Determinante elektoralen Erfolgs (Mitchell 2000, S. 341). Parteiunabhängige Strategien haben keinen Wert bzw. können sich sogar schädlich auf die politische Karriere auswirken. Als klassisches - obgleich empirisch seltenes ${ }^{5}$ - Beispiel gelten reine Verhältniswahlsysteme mit geschlossenen Listen, in denen die Parteiführung über die Vergabe der Listenplätze bestimmt. Der individuelle Wahlerfolg ist hier vollständig vom Stimmenanteil der Partei und dem eigenen Platz auf der Wahlliste abhängig.

Zweifellos ist auch in kandidatenzentrierten Wahlsystemen die Parteimitgliedschaft eines Bewerbers zentral für seine Erfolgsaussichten bei der Wahl. Dies liegt zum Teil daran, dass die Wähler das in einigen Wahlsystemen angelegte Potenzial gezielter Personenwahl nur eingeschränkt nutzen (z. B. Schoen u. Faas 2005). Wie verschiedene empirische Befunde zeigen (z. B. Stratmann u. Baur 2002; Lancaster u. Patterson 1990; Zittel u. Gschwend 2007; Sieberer 2010; Zittel 2008; Patzelt 2007), sollte der Einfluss der personal vote auf Rollenverständnis und Verhalten von Abgeordneten jedoch nicht unterschätzt werden (Klingemann u. Wessels 2003). Entsprechend gilt es auch in den Ländern zu prüfen, ob das Wahlsystem die Dissensneigung von Abgeordneten beeinflusst. ${ }^{6}$

\section{H1: Je stärker kandidatenzentriert das Wablsystem ausgestaltet ist, desto geringer sollte die Fraktionsgeschlossenheit ausfallen.}

Wie sich die Beteiligung an der Regierung auf das Abstimmungsverhalten auswirkt, wird in der bestehenden Forschung widersprüchlich beurteilt. Regierungsfraktionen wird einerseits eine höhere Geschlossenheit als Oppositionsfraktionen vorausgesagt. Durch die Übernahme der Regierung fielen der Mehrheitsfraktion mehr institutionelle Mittel zur Sicherstellung von Geschlossenheit zu (z. B. durch die Vertrauensfrage) und ihre Abgeordneten binde die Aufgabe, die Regierung im Amt zu halten (Carey 2007, S. 95; Davidson-Schmich 2003). Der Zusammenhang könnte jedoch auch in die andere Richtung wirken. Der Regierungsstatus zwingt Fraktionen häufig zur Abstimmung über unpopuläre Maßnahmen und insbesondere bei Koalitionen zu teils schmerzhaften Kompromissen (Sieberer 2006b, S. 157; Depauw u. Martin 2008). In diesem Sinne verbessert der Regierungsstatus die Chancen für Geschlossenheit auf der Disziplinierungsseite, verschlechtert sie jedoch auf Ebene der Kohäsion. Im Endeffekt erscheint es durchaus möglich, dass sich beide Komponenten gegenseitig aufheben und empirisch kein Zusammenhang zwischen Regierungsstatus und Fraktionsgeschlossenheit nachweisbar ist. Dennoch ist aus der Grundlogik parlamentarischer Regierungssysteme die Erwartung ableitbar, dass Regierungsfraktionen geschlossener auftreten sollten. Zumindest Abstimmungen über wichtige Vorlagen sind implizite Vertrauensabstimmungen, sodass das Überleben der Regierung von ihrer hinreichenden Geschlossenheit abhängt. Es sollte also gelten:

5 Diese Macht der Fraktionsführung ist sehr selten (Lundell 2004, S. 36), in Deutschland praktisch nicht zu finden (Patzelt 2006, S. 112; Schüttemeyer u. Sturm 2005).

6 Es existieren keine hinreichenden Daten zu Kandidatennominierungsverfahren auf Länderebene, sodass hier nur wahlsystemische Anreize berücksichtigt werden können. 


\section{H2: Regierungsfraktionen sind geschlossener als Oppositionsfraktionen.}

Auch die parlamentarischen Mehrheitsverhältnisse stehen in einem ursächlichen Zusammenhang mit dem Abstimmungsverhalten. Fraktionen können umso weniger Dissens tolerieren, desto knapper die Mehrheitsverhältnisse im Parlament sind. Insbesondere für die Regierungsfraktion ist nun jede Stimme notwendig, um Abstimmungen zu gewinnen (Bowler et al. 1999, S. 14). In abgeschwächter Form gilt dies auch für die Opposition. Durch hohe Geschlossenheit zwingt sie die Regierung ihrerseits zur Geschlossenheit, da bei dem üblichen Erfordernis einer einfachen Mehrheit jeder oppositionelle Abweichler die Regierungsfraktion entlastet. Zudem wahrt die Opposition die minimale Chance, eine Abstimmung zu gewinnen.

Übergroße Regierungsmehrheiten verringern hingegen die Kosten des Abweichens, da die Handlungsfähigkeit der Regierung nicht unmittelbar gefährdet wird. In diesem Fall braucht die Fraktionsführung nur so viele Stimmen zu sichern, wie zum Gewinn einer Abstimmung nötig sind (Cox u. McCubbins 2005, S. 218). Durch die zunehmende Größe einer Fraktion stehen zudem relativ weniger Ämter zur Verfügung, die als einer der wirkungsvollsten Anreize für loyales Verhalten gelten (Benedetto u. Hix 2007).

\section{H3: Je knapper die Regierungsmehrheit, desto höher die Fraktionsgeschlos- senheit.}

Ein zentraler Faktor für die Geschlossenheit von Parlamentsfraktionen ist die Verteilung der politischen Präferenzen - bei Hazan im Begriff „cohesion“ zusammengefasst. Idealbedingungen für Geschlossenheit bilden eine hohe innerparteiliche Homogenität und zwischenparteiliche Heterogenität der Präferenzen (Krehbiel 1999). Mit anderen Worten: Je stärker die Fraktionsmitglieder ähnliche politische Einstellungen teilen und sich dabei von den Einstellungen anderer Fraktionen unterscheiden, desto seltener sind sie versucht, mit anderen Fraktion zu stimmen oder gar vollständig auf deren Bänke zu wechseln (Bowler et al. 1999, S. 6; Owens 2006, S. 13; Saalfeld 2005, S. 44-49). In einem Zweiparteiensystem sollte folgerichtig mit steigender politischer Distanz zwischen den Fraktionen ihre Geschlossenheit zunehmen. In den in den deutschen Ländern vorherrschenden Mehrparteiensystemen mit Koalitionsregierung muss eine Hypothese komplexer ausfallen. Eine wachsende Distanz zwischen den Fraktionen wäre beispielsweise für die Geschlossenheit von Koalitionen eher eine ,Hypothek' (Martin u. Vanberg 2004; Miller 2007). Da in der parlamentarischen Praxis bei Abstimmungen häufig Regierungsentwürfe mit oppositionellen Alternativentwürfen ${ }^{7}$ verglichen werden, ist es sinnvoll, die Distanz zwischen Regierung und Opposition als Prädiktor der Fraktionsgeschlossenheit heranzuziehen.

H4: Mit steigender politischer Distanz zwischen Regierung und Opposition sollte die Fraktionsgeschlossenheit zunehmen.

7 Dieser Vergleich kann durchaus implizit bleiben oder in Form von Änderungsanträgen zu Regierungsgesetzen explizit sein. 
Namentliche Abstimmungen bzw. daraus abgeleitete Maße wie der Rice-Index (Rice 1928) dienen als zentraler Indikator für die Geschlossenheitsforschung. Wie diese Datenbasis entsteht, wird meist nicht genauer hinterfragt, ihre Repräsentativität häufig per Annahme „sichergestellt“ (z. B. Hix 2002, S. 629). Insbesondere wird in Untersuchungen zur Geschlossenheit nicht überprüft, ob der Auswahlprozess namentlicher Abstimmungen aus dem Universum aller Abstimmungen zu einer systematischen Verzerrung der Geschlossenheitswerte führen kann. In Parlamenten, wie dem U.S. Kongress, wo roll call votes standardmäßig verwendet werden, braucht man sich über diese Fragen selbstredend auch nicht den Kopf zu zerbrechen (aber siehe Clinton 2007). Virulent werden diese methodologischen Probleme jedoch, wenn - wie in den meisten Parlamenten (Hug 2010; Saalfeld 1995a) - namentliche Abstimmungen nur auf Verlangen einer Fraktion zur Anwendung kommen. Das dabei zum Tragen kommende Handlungskalkül, so zeigen verschiedene jüngere Beiträge, kann die Datenbasis systematisch verzerren und zwar genau entlang der Dimension, die damit untersucht werden soll: Fraktionsgeschlossenheit bzw. der Kohäsion von Fraktionen als eine Bedingung ihres Abstimmungsverhaltens.

In gebotener Kürze (ausführlich: Carrubba et al. 2006; Stecker 2010; Thiem 2006) soll dieses Kalkül und die Konsequenz für das Datensample hier skizziert werden. Hinter einem Antrag auf namentliche Abstimmung stehen überwiegend zwei Ziele (siehe zu einer allgemeinen Diskussion Saalfeld 1995a, S. 554-559): öffentlich Farbe zu einer politischen Frage zu bekennen („position-taking“) oder das Abstimmungsverhalten eines politischen Wettbewerbers im Parlament offenzulegen bzw. sich davon abzugrenzen („,negatives position-taking“). Die Überwachung und Disziplinierung eigener Hinterbänkler über namentliche Abstimmungen spielt dagegen eine untergeordnete Rolle. Sie ist zum einen schlicht unnötig, da in den übersichtlichen Landtagen eine visuelle Kontrolle bei halb-offenen Abstimmungen hinreichend sein sollte. Zum anderen birgt sie sogar Risiken. Namentliche Abstimmungen liefern Informationen über individuelles Verhalten auch an competing principals wie Wahlkreise und Interessengruppen. Diese werden damit in die Lage versetzt, einzelne Abgeordnete zu kontrollieren und gegebenenfalls deren Verhalten zu beeinflussen - eine Möglichkeit, die bei den standardmäßigen halboffenen Abstimmungen kaum besteht. In den USA ist die gezielte parteiunabhängige Unterstützung einzelner Kandidaten durch Interessengruppen auf Basis des individuellen Abstimmungsverhaltens gängige Praxis (Bell u. Roberts 2005). Sitzen also einigen Regierungsabgeordneten competing principals mit von der Fraktionsführung abweichenden Interessen ,im Nacken', dürfte ein Antrag auf namentliche Abstimmung die Geschlossenheit eher gefährden. Eine rationale Regierungsfraktionsführung wird also darauf verzichten, während Oppositionsfraktionen ausgiebig davon Gebrauch machen dürften. Die Rhetorik in den Plenarprotokollen (Stecker 2011) und Interviews mit Landtagsparlamentariern (Könen 2009) unterstützen diese Vermutung.

Daraus folgt, dass Fraktionen den Namensaufruf meist bei Beschlussgegenständen verlangen, die ihre Mitglieder unisono unterstützen bzw. über die politische Wettbewerber intern zerstritten sind. Ein Beispiel: Sieht sich die Regierung angesichts knapper Kassen zu einer unpopulären Kürzungspolitik gezwungen, wird dies 
in ihren eigenen Reihen nicht unumstritten sein. Abgeordnete aus nachteilig betroffenen Wahlkreisen etwa, werden sich nur durch ,discipline' zu einer Zustimmung bewegen lassen. Beantragt die Opposition eine namentliche Abstimmung, kann sie sich dadurch öffentlichkeitswirksam gegen die Maßnahme positionieren und zusätzlich die unbeliebte Position der Regierungsfraktion oder gar ihre interne Zerstrittenheit offen legen. Im Jargon der Geschlossenheitsforschung zusammengefasst: Namentliche Abstimmungen werden von Fraktionen beantragt, die von kohäsionsinduzierter Geschlossenheit in ihren Reihen ausgehen können, bzw. zu Vorlagen beantragt, bei denen interne Präferenzheterogenität die Geschlossenheit der Wettbewerber gefährdet. Entsprechend ihrer höheren Kohäsion sollte die beantragende Fraktion ceteris paribus auch höhere Geschlossenheitswerte aufweisen. Also:

H5: Die Fraktion, die eine namentliche Abstimmung beantragt, sollte ge-
schlossener abstimmen.

\section{Forschungsdesign}

Die diskutierten Hypothesen werden mit Daten zu allen 2184 namentlichen Abstimmungen aus insgesamt 42 Wahlperioden aller 16 bundesdeutschen Länderparlamente überprüft. Die Bundesländer bieten für die vorliegende Fragestellung ein denkbar günstiges Verhältnis zwischen experimenteller Varianz und der Kontrolle externer Störgrößen (siehe zu ihrem Potenzial für vergleichende Forschung auch Kropp 2001; Jun et al. 2008; Bräuninger u. Debus 2008; Flick u. Vatter 2007; Müller 2009). Insbesondere die Probleme international vergleichender Forschung, die u. a. aus variierenden Regelungen zu namentlichen Abstimmungen resultieren (Hug 2006, S. 24-34; Owens 2006, S. 15) treten hier nicht auf. In den Ländern bestehen präzise und weitgehend identische geschäftsordnungsrechtliche Regelungen über den Namensaufruf. Demnach hat jede Fraktion oder eine äquivalente Anzahl von Abgeordneten ${ }^{8}$ das Recht, eine namentliche Abstimmung zu einem substanziellen Beschlussgegenstand (Gesetze, Änderungsanträge etc.) zu beantragen. Gleichzeitig bieten die Länderparlamente die Bedingungen eines crucial Tests. Die informellen Anreize zur Geschlossenheit sind in Deutschland extrem hoch (Patzelt 1998; Patzelt 2006). Mutmaßlich reichte der durch soziologische Faktoren erzeugte Zusammenhalt allein aus, um eine hohe Geschlossenheit zu erzielen. Gelingt es in diesem Umfeld dennoch nachzuweisen, dass bestimmte institutionelle Variablen einen Einfluss entfalten, dürfen diese Effekte als relativ robust gelten.

Um die Geschlossenheitswerte zu erhalten, wurden zunächst die Abstimmungslisten manuell aus den amtlichen Plenarprotokollen extrahiert. ${ }^{9}$ Diese Listen wurden mit Hilfe einer Software zur Mustererkennung regulärer Ausdrücke halb-automatisch in ein für das Statistikprogramm Stata lesbares Format überführt. Nun wurde für jede Abstimmung und Partei die Geschlossenheit errechnet. Die Präsen-

8 In Schleswig-Holstein bedarf es für einen Antrag auf namentliche Abstimmung allerdings $18 \mathrm{Ab}-$ geordneter, was etwa einem Sitzanteil von $26 \%$ entspricht.

9 Die Protokolle wurden von den Internetpräsenzen der Landtage oder von www.parlamentsspiegel. de bezogen. Die Verfügbarkeit von Plenarprotokollen, die mit vertretbarem Aufwand auswertbar waren, variiert sehr stark. Daher sind einzelne Länder unterschiedlich häufig vertreten (siehe Tabelle 1). 
tation der Ergebnisse erfolgt mit Hilfe des Index of Agreement, einer Modifikation des bekannteren Rice-Index, die auch Enthaltungen berücksichtigt.

$$
A I_{i}=\frac{\max \left\{J_{i}, N_{i}, E_{i}\right\}-\frac{1}{2}\left[\left(J_{i}+N_{i}+E_{i}\right)-\max \left\{J_{i}, N_{i}, E_{i}\right\}\right]}{\left(J_{i}+N_{i}+E_{i}\right)}
$$

„J“ bzw. „N“ bzw. „E“ beziffern in der Formel dabei die Ja- bzw. Neinstimmen bzw. die Enthaltungen einer Fraktion bei einer Abstimmung i. Alle Abgeordneten, die gegen die Mehrheit der Fraktion stimmen, sei es mit „Ja“, „Nein“ oder „Enthaltung“, gehen als gleichgewichtete Abweichler in die Berechnung ein. Entsprechend nimmt der Agreement-Index den Wert „1“ an, wenn sich alle Fraktionsmitglieder entweder geschlossen enthalten oder mit "Ja“ bzw. „Nein“ stimmen. "0" Einigkeit herrscht dagegen, wenn eine Fraktion wie auch immer gleichmäßig über diese Kategorien verteilt ist. Mit der Berücksichtigung von Enthaltungen wird anerkannt, dass auch sie eine Form abweichenden Stimmverhaltens darstellen (Saalfeld 1995b, S. 73-77). Abwesenheiten mussten leider ignoriert werden, da es empirisch im Einzelfall nicht möglich ist, ihre Bedeutung in Bezug auf Fraktionsgeschlossenheit zu interpretieren (dagegen Carey 2007).

Tabelle 1: Übersicht über den Datensatz

\begin{tabular}{lcccc}
\hline Bundesland & Beginn & Ende & Anzahl WP & RCV \\
\hline Brandenburg & $10 / 1990$ & $08 / 2009$ & 4 & 315 \\
Berlin & $11 / 1995$ & $08 / 2006$ & 3 & 90 \\
Baden-Württemberg & $06 / 1988$ & $10 / 2011$ & 4 & 188 \\
Bayern & $10 / 1994$ & $07 / 2008$ & 3 & 535 \\
Bremen & $07 / 1995$ & $04 / 2007$ & 3 & 13 \\
Hessen & $04 / 1999$ & $12 / 2007$ & 2 & 18 \\
Hamburg & $10 / 2001$ & $02 / 2011$ & 3 & 29 \\
Mecklenburg-Vorpommern & $10 / 1998$ & $06 / 2006$ & 2 & 37 \\
Niedersachsen & $03 / 2003$ & $01 / 2008$ & 1 & 5 \\
Nordrhein-Westfalen & $06 / 2000$ & $03 / 2010$ & 2 & 40 \\
Rheinland-Pfalz & $05 / 2001$ & $02 / 2006$ & 1 & 8 \\
Schleswig-Holstein & $04 / 1996$ & $01 / 2005$ & 2 & 15 \\
Saarland & $09 / 1999$ & $01 / 2009$ & 2 & 8 \\
Sachsen & $10 / 1990$ & $06 / 2004$ & 3 & 108 \\
Sachsen-Anhalt & $07 / 1994$ & $02 / 2006$ & 3 & 127 \\
Thüringen & $10 / 1990$ & $10 / 2008$ & 4 & 648 \\
\hline & & 184 Jahre & 42 & 2184 \\
\hline
\end{tabular}

Quelle: eigene Auswertung. 
Tabelle 1 fasst den Datensatz zusammen. Insgesamt stehen für die Analyse 2184 namentliche Abstimmungen (RCV) aus 42 Wahlperioden zur Verfügung. Die folgende Auswertung konzentriert sich auf die wichtigsten Fraktionen CDU/CSU, SPD, B'90/Die Grünen, FDP und Linkspartei bzw. PDS. ${ }^{10}$ Damit ergeben sich 8120 Geschlossenheitswerte. Dabei variiert die Häufigkeit namentlicher Abstimmungen über die Länder hinweg sehr stark. Während für die Hälfte der Landtage nur sehr wenige Abstimmungen vorliegen, liefern die ostdeutschen Parlamente sowie Bayern und Baden-Württemberg eine wesentlich größere Anzahl (zu einer Erklärung dieser variierenden Häufigkeit: Stecker 2010). Betrachtet man genauer, wer die namentlichen Abstimmungen beantragt hat, ergibt sich über alle Länder hinweg ein eindeutiges Bild: Zu durchschnittlich 80 Prozent erfolgt der Namensaufruf auf Verlangen einer Oppositionsfraktion. In nur knapp einem Zehntel der Fälle geht er auf eine Regierungsfraktion zurück. Die übrigen zehn Prozent ergeben sich direkt aus der Abstimmungsmaterie, z. B. bei Verfassungsänderungen, die namentlich abgestimmt werden müssen. Diese Werte sprechen dafür, dass das oben skizzierte Kalkül für einen Großteil der Anträge auf namentliche Abstimmungen verantwortlich ist. Entsprechend muss bedacht werden, dass damit ein klarer Rahmen für die Interpretation der folgenden Ergebnisse gesetzt wird. Diese werfen ihr Licht vorrangig auf eine ganz spezifische Abstimmungssituation: Eine kohäsive (Oppositions-)Fraktion will ihre Position sichtbar verdeutlichen bzw. Zerstrittenheit in anderen - meist den Regierungs- - Fraktionen aufdecken oder diese zwingen, öffentlich zu einer eventuell unpopulären Maßnahme zu stehen.

Für die empirische Analyse wurden alle 28 Abstimmungen aussortiert, in denen vollständiger Konsens im Parlament herrschte. Diese Abstimmungen stehen offensichtlich außerhalb des parteipolitischen Konfliktes und würden die Geschlossenheit überschätzen. ${ }^{11}$ Aus einem ähnlichen Grund sollten Abstimmungen ausgeschlossen werden, die von der Fraktionsführung freigegeben wurden. Leider war es nicht mit vertretbarem Aufwand möglich, diese - in der parlamentarischen Praxis vermutlich äußerst seltenen - Abstimmungen zu identifizieren. ${ }^{12}$

Die unabhängigen Variablen werden folgendermaßen operationalisiert. Die Messung wahlsystemischer Anreize folgt dem Vorschlag von Norris (2004), die vereinfacht in parteizentrierte und kandidatenzentrierte Wahlsysteme sowie eine Zwischengruppe unterscheidet (vgl. Massicotte 2003). Bayern und Baden-Württemberg zähle ich zu den kandidatenzentrierten Wahlsystemen. In Bayern ermöglicht die Einerwahl in 92 Stimmkreisen und eine offene Liste in sieben Wahlkreisen die gezielte Wahl einzelner Kandidaten. Auch in Baden-Württemberg sind die Wahlchancen der Parlamentarier insbesondere von der Unterstützung im Wahlkreis abhängig (Riescher u. Gebauer 2004, S. 63; Müller 2004, S. 294), was zu

10 Auch die Partei Rechtsstaatliche Offensive (PRO) ist aufgrund ihrer Regierungsbeteiligung in Hamburg in die Analyse eingeschlossen.

11 Dabei kann die Erklärung des Ausmaßes an Konsens (legislative inclusiveness) selbst interessant sein (Ganghof 2010).

12 Diese Information war nicht aus den Plenarprotokollen ersichtlich. Theoretisch ließe sich anhand des Votums des engeren Fraktionsvorstandes die Freigabe einer Abstimmung ableiten. Stimmte der Fraktionsvorstand uneinheitlich, dürfte dies meist auf das Fehlen einer klaren Vorgabe hindeuten (Snyder u. Groseclose 2000). 
einer stärkeren Unabhängigkeit der Abgeordneten von ihrer Fraktion führen dürfte. Hamburg, Bremen und das Saarland, die mit minimalen Unterschieden ein einfaches Verhältniswahlrecht mit starren Listen anwenden, werden als parteizentrierte Systeme klassifiziert. Die größte Gruppe bilden die elf übrigen Länder mit gemischten Wahlsystemen. Die unterschiedlichen Anteile von Direkt- und Listenmandataren ${ }^{13}$ in diesen Parlamenten bieten sich auf den ersten Blick für eine feinere Binnenoperationalisierung elektoraler Anreize an. Da wir jedoch zu wenig über die Kontaminationseffekte zwischen unterschiedlichen Mandatstypen in gemischten Wahlsystemen wissen (Bawn u. Thies 2003; Crisp 2007), verzichte ich auf diese Möglichkeit.

Die Regierungsmehrheit ist als die logarithmierte Anzahl der Sitze gemessen, die die Regierung über das absolute Mehrheitserfordernis hinaus kontrolliert. Hinter dem Logarithmieren steht die Überlegung, dass sich der dissensbegünstigende Effekt der Mehrheitsgröße mit jedem zusätzlichen Sitz abschwächt. Die politische Distanz zwischen Regierung und Opposition wird mithilfe der von Bräuninger und Debus (2008) aus den Länderparteiprogrammen gewonnenen Positionsdaten auf einer Links-Rechts-Achse berechnet. Zweifellos wäre es exakter im Hinblick auf Politikfeld und Beschlussvorlage spezifische Positionen für jede Abstimmung zu verwenden. Leider existieren keine derartigen Daten. Sofern keine Einparteienregierung vorliegt, wird die Regierungsposition aus der mit dem Sitzanteil gewichteten mittleren Position aller Koalitionspartner operationalisiert. Analog erfolgt die Bestimmung der Position der Opposition. In den statistischen Test geht die absolute Differenz beider Werte ein. Leider existieren zum jetzigen Zeitpunkt keine Daten zur internen Präferenzhomogenität einzelner Fraktionen in den Länderparlamenten, die notwendig wären, um den Einfluss der Verteilung politischer Präferenzen vollständig zu überprüfen (Pappi u. Seher 2009). ${ }^{14}$ Die Information, welche Fraktion eine namentliche Abstimmung beantragt hat, wurde im Einzelnen aus den Plenarprotokollen erhoben und entsprechend kodiert.

\section{Empirische Analyse}

Die Abbildungen 1 und 2 präsentieren deskriptive Informationen über die Fraktionsgeschlossenheit in den Landtagen: Die gruppierten Boxplots zeigen die Geschlossenheitswerte aller Fraktionen über alle Abstimmungen in den einzelnen Bundesländern, das Punktediagramm trägt den Mittelwert der Geschlossenheit nach verschiedenen Kategorien ab. Die Boxplots unterstreichen eindrucksvoll, dass perfekte Geschlossenheit in den deutschen Länderparlamenten die Norm ist. Der mediane Geschlossenheitswert einer Fraktion bei einer Abstimmung liegt bei 100 Prozent. In einigen Ländern betrifft dies sogar 75 Prozent der Geschlossenheitswerte. ${ }^{15}$ In Niedersachsen und Schleswig-Holstein sind alle Fraktionen bei

13 Das Verhältnis von Listen- zu Direktmandataren variiert zwischen Niedersachen $(1: 1,8)$ und Sachsen-Anhalt (1:1).

14 Die vom SFB580 in Jena (z. B. Best u. Jahr 2006) erhobenen individuellen Positionsdaten liegen nur für wenige Wahlperioden vor.

15 Die vertikale Linie in einem Boxplot repräsentiert den Median, die Box schließt 75 \% der Werte ein und die einzelnen Punkte stehen für Ausreißer. 
jeder Abstimmung perfekt geschlossen. Allerdings kommt es darüber hinaus auch zu nennenswerten Abweichungen, sehr selten sogar zu vollständigen Zusammenbrüchen der Fraktionsgeschlossenheit. Deutlicher Dissens tritt gelegentlich in Bayern und Baden-Württemberg, vor allem aber in den fünf ostdeutschen Landtagen auf. Dort ergeben sich niedrige Geschlossenheitswerte besonders häufig in den ersten beiden Wahlperioden (vgl. auch Davidson-Schmich 2006; Könen 2009). Erst in ihrer dritten und vierten Wahlperiode erreichen die Fraktionen in den neuen Ländern Geschlossenheitswerte auf westdeutschem Niveau. ${ }^{16}$

Direkte materielle Konsequenzen zeitigt auftretender Dissens allerdings so gut wie nie. Regierungsfraktionen votieren fast immer hinreichend einig, um Abstimmungen zu gewinnen. In nur 18 Fällen, (0,8 Prozent aller Abstimmungen) verliert die Regierung aufgrund mangelnder Geschlossenheit. Darunter finden sich aber zehn Fälle von mangelnder Koalitionsgeschlossenheit (insbesondere bei der großen Koalition in Berlin), das heißt, beide oder mehrere Regierungspartner stimmen - weitgehend geschlossen - gegeneinander.

Das Punktediagramm erlaubt es darüber hinaus, den Einfluss verschiedener Faktoren auf die Fraktionsgeschlossenheit zu beurteilen. Die vertikale gestrichelte Linie repräsentiert dabei den Gesamtmittelwert von 0,955. Diesen Wert erhält beispielsweise eine 31-köpfige Fraktion, in der ein Abgeordneter abweicht. Es zeigt sich wie erwartet, dass eine höhere Geschlossenheit resultiert, wenn eine Fraktion an der Regierung beteiligt ist oder die entsprechende namentliche Abstimmung beantragt hat. Überdurchschnittlich große Regierungsmehrheiten begünstigen hingegen häufigeren Dissens. Die Kandidatenzentrierung des Wahlsystems zeigt zumindest in dieser bivariaten Betrachtung nicht exakt den postulierten Effekt. Der bereits in den Boxplots aufscheinende Unterschied zwischen Ost- und Westdeutschland ist auch im Punktediagramm prononciert. Allerdings können diese Mittelwerte nur einen ersten Eindruck vermitteln, da nicht für die Einflüsse der anderen Variablen kontrolliert wird. Beispielsweise wird der Geschlossenheitswert in gemischten Wahlsystemen dadurch nach unten gezogen, dass alle ostdeutschen Länder dieses System praktizieren. Eine sicherere Schätzung der Effekte soll im Folgenden ein multivariater Test liefern.

16 Die Auswertungen von Davidson-Schmich (2006) zeigen, dass die Geschlossenheit in westdeutschen Landtagen auch in den frühen 1990er Jahren sehr hoch war. 
Abbildung 1: Geschlossenheit von CDU/CSU, SPD, Grüne, FDP und Linkspartei bei jeder Abstimmung nach Bundesländern

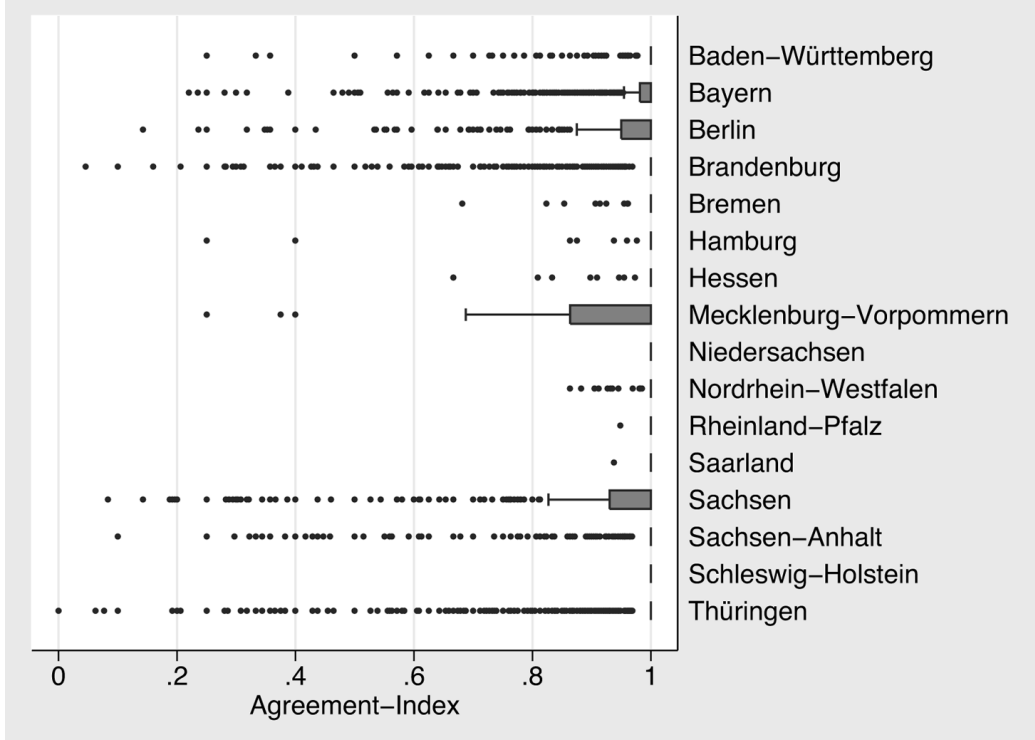

Quelle: eigene Berechnung.

Abbildung 2: Durchschnittliche Geschlossenheitswerte nach verschiedenen unabhängigen Variablen



Quelle: eigene Berechnung. 
Dabei müssen zwei in der Datenstruktur liegende Besonderheiten berücksichtigt werden. Erstens postulieren die diskutierten Hypothesen einen Einfluss verschiedener Faktoren auf vier verschiedenen Ebenen: das Wahlsystem auf Systemebene, Regierungsmehrheit und politische Distanz auf Parlamentsebene, Regierungsstatus auf Fraktionsebene sowie schließlich die Beantragung des Namensaufrufs auf Abstimmungsebene. Diese Ordnung scheint nach einer Mehrebenenanalyse zu verlangen. Insbesondere die geringe Fallzahl von nur 16 Ländern, die zudem keine Zufallsauswahl darstellt, sowie die nicht stringent hierarchische Struktur der Daten, schließen diese Möglichkeit jedoch aus (vgl. Braun et al. 2010, S. 20-22).

Zweitens produziert der Index of Agreement nur Werte, die zwischen 0 und 100 liegen. ${ }^{17}$ Die Determinanten einer solchen limited dependent variable lassen sich streng genommen nicht mittels eines einfachen Regressionsmodells schätzen, da dieses unter anderem auf der Annahme basiert, dass sich die abhängige Variable in einem unendlich großen Wertebereich bewegt. Ein angemesseneres Schätzverfahren bieten in diesem Fall sogenannte Fractional Response-Modelle (Long u. Freese 2006). Aufgrund der besseren Interpretierbarkeit und um die Vergleichbarkeit mit weiteren Beiträgen zu vereinfachen (Depauw u. Martin 2008; Carey 2007), erfolgt die Präsentation der Ergebnisse dennoch mit einem Regressionsmodell. ${ }^{18}$

Die Variablen Regierungsmehrheit und politische Distanz gehen in der beschriebenen Operationalisierung in die Regression ein. Zur Bestimmung des Effekts gemischter und kandidatenzentrierter Wahlsysteme werden Dummy-Variablen in das Regressionsmodell aufgenommen, wobei parteizentrierte Wahlsysteme als Referenzkategorie dienen. Wie bereits die deskriptive Auswertung zeigt, weisen die Fraktionen in ostdeutschen Landtagen wesentlich niedrigere Geschlossenheitswerte auf. Dies ist allerdings nicht institutionellen Effekten geschuldet, sondern den Einstellungen und dem Amtsverständnis der Nachwendeparlamentarier. Sie bezeugten eine prinzipiell höhere Responsivitätsbereitschaft gegenüber den Bürgern (Patzelt 1996, S. 469) und standen Fraktionsgeschlossenheit nach den Erfahrungen in der DDR misstrauisch gegenüber (Davidson-Schmich 2006, S. 88; Könen 2009). Da diese soziologischen Faktoren geeignet sind, die Analyse der institutionellen Effekte zu verzerren, wird für die ersten beiden Wahlperioden in Ostdeutschland mit einer Dummy-Variable kontrolliert. ${ }^{19}$ Um der wechselseitigen Abhängigkeit der Beobachtungseinheiten Rechnung zu tragen, werden die Standardfehler auf Ebene der Abstimmungen geclustert.

Insgesamt werden zwei Modelle berechnet. Modell 1 enthält alle Variablen aller vier Ebenen, während in Modell 2 die Systemvariable Wahlsystem durch Länder-Dummies ersetzt wird. Modell 2 erlaubt es damit, die Robustheit der unter

17 Zur besseren Lesbarkeit der Ergebnisse wurde der ursprüngliche Index für die Regression mit 100 multipliziert.

18 Unter Verwendung eines fractional response models ergeben sich keine substanziellen Veränderungen. Die Ergebnisse können vom Verfasser bezogen werden.

19 Aufgrund der x-zentrierten Kausalperspektive besitzt dieser Faktor nicht den Status einer erklärenden, sondern einer Kontrollvariable (Sieberer 2007). 
der Systemebene angesiedelten Effekte abzusichern. Tabelle 2 enthält die Ergebnisse der multivariaten Regression. ${ }^{20}$

In Modell 1 zeigt sich eindeutig, dass entgegen H1 zunehmende Personal-VoteAnreize keine konsistente Minderung der Fraktionsgeschlossenheit herbeiführen. Zwar senken gemischte gegenüber parteizentrierten Wahlsystemen die Geschlossenheit. In kandidatenzentrierten Systemen herrscht allerdings die höchste Einigkeit - auch wenn der Effekt nicht signifikant ist. ${ }^{21}$ Bei einer Regression ohne die ostdeutschen Landtage ist auch die Wirkung gemischter Wahlsysteme nicht mehr von Null zu unterscheiden.

Wie in $\mathrm{H} 2$ postuliert, erhöht die Beteiligung an der Regierung die Fraktionsgeschlossenheit. Regierungsfraktionen erzielen eine um durchschnittlich 2,4 Punkte höhere Einigkeit als Oppositionsfraktionen. Dieses Ergebnis ist über beide Modelle hinweg stabil. Alle Fraktionen scheinen zudem mehr Dissens zu tolerieren, wenn die Regierung von einer komfortablen Mehrheit zehren kann. Werden Dummy-Variablen für die Bundesländer statt der Wahlsystem-Variablen berücksichtigt (Modell 2), verschwindet dieser Effekt. Dies ist auf die Unterschiedlichkeit der Parteiensysteme der Bundesländer zurückzuführen, die mit unterschiedlichen Koalitionen und Parlamentsmehrheiten einhergeht. Von dem vergleichsweise deutlichsten Geschlossenheitsbonus kann die Fraktion profitieren, die die entsprechende namentliche Abstimmung beantragt hat. Sie weist einen um fast 5 bzw. 4,6 Punkte höheren Wert des Index of Agreement auf. Bei der äußerst geringen Varianz um einen sehr hohen Mittelwert von 95 kann dies als sehr starker Wirkungsfaktor betrachtet werden. Eine zunehmende ideologische Distanz zwischen Opposition und Regierung senkt den fraktionsinternen Dissens. Dieser Effekt ist allerdings bei beiden Modellen äußerst dezent. Mit jedem weiteren Punkt, den sich Regierung und Opposition auf der zwischen 0 und 10 variierenden RechtsLinks-Achse voneinander entfernen, steigt der Index of Agreement nur um knapp 0,1 Punkt. Der Koeffizient der Dummy-Variablen für die ersten beiden Wahlperioden in den ostdeutschen Landtagen bestätigt erwartungsgemäß die stärkere Dissensneigung der ostdeutschen Nachwendeparlamentarier. Repliziert man beide Modelle mit dem klassischen Rice-Index ${ }^{22}$, dessen Korrektur durch Desposato ${ }^{23}$ oder der Modifikation von Saalfeld (1995b), bei dem Enthaltungen als mit 0,5 gewichtete Abweichungen eingehen, bleiben Richtung und Signifikanz der Koeffizienten weitgehend unverändert.

20 Aufgrund von einigen fehlenden Informationen zum Antragsteller einer namentlichen Abstimmung stehen 5970 Geschlossenheitswerte zur Verfügung.

21 Broscheid und Gschwend (2005) argumentieren überzeugend, dass wir Stochastizität immer berücksichtigen sollten, wenn wir allgemeine Erklärungen über eine komplexe soziale Realität testen. Entsprechend berücksichtige ich hier Signifikanzwerte und Konfidenzintervalle, auch wenn die zu Grunde liegenden Daten als Vollerhebung aufgefasst werden können.

22 Der Rice-Index erfasst die absolute Differenz zwischen dem Anteil der Ja- und Neinstimmen einer Fraktion und setzt sie zur Gesamtheit der mit "Ja" und „Nein“ stimmenden Fraktionsmitglieder ins Verhältnis. Enthaltungen werden ignoriert. Mit anderen Indizes errechnete Ergebnisse sind beim Verfasser erhältlich.

23 Desposato (2005) hat darauf aufmerksam gemacht, dass die gebräuchlichen Geschlossenheitsindizes die Werte kleinerer Fraktionen systematisch überschätzen und dafür eine mathematische Korrekturmöglichkeit erarbeitet. 
Tabelle 2: Ergebnisse der OLS-Regression. Abhängige Variable: Fraktionsgeschlossenheit (Agreement-Index) einer Fraktion bei einer Abstimmung

\begin{tabular}{lcc}
\hline Unabhängige Variablen & $\begin{array}{c}\text { Modell 1 } \\
\text { (volles Modell) }\end{array}$ & $\begin{array}{c}\text { Modell 2 } \\
\text { (fixed effects) }\end{array}$ \\
\hline Gemischtes WS & $-1.988^{* *}$ & - \\
Kandidatenzentriertes WS & $(0.699)$ & - \\
Regierungsbeteiligung & 0.887 & $1.928^{* * *}$ \\
& $(0.683)$ & $(0.371)$ \\
Mehrheitsgröße & $2.352^{* * *}$ & 0.220 \\
& $(0.373)$ & $(0.297)$ \\
RCV-Antrag & $-0.592^{* *}$ & $4.621^{* * *}$ \\
Ideologische Distanz & $(0.200)$ & $(0.343)$ \\
& $4.929 * * *$ & $0.103 *$ \\
Ostdeutschland bis WP2 & $(0.339)$ & $(0.0480)$ \\
Länder-Dummies & $0.120^{* * *}$ & $-3.387^{* * * *}$ \\
Konstante & $(0.0419)$ & $(0.820)$ \\
& $-3.803^{* * *}$ & nicht \\
Beobachtungen & $(0.627)$ & abgebildet \\
Korrigiertes $R^{2}$ & - & $92.08^{* * * *}$ \\
\hline
\end{tabular}

Anmerkungen: robuste Standardfehler in Klammern.

${ }^{*}=p<0,05 ;{ }^{* *}=p<0,01 ;{ }^{* * *}=p<0,001$.

\section{Schlussbetrachtung}

Der vorliegende Beitrag hat anhand von 2184 namentlichen Abstimmungen aus insgesamt 42 Wahlperioden seit 1990 die Fraktionsgeschlossenheit in allen 16 Länderparlamenten analysiert und dabei die Wirkung prominenter Faktoren getestet.

Als zentraler deskriptiver Befund kann festgehalten werden, dass in den Landtagen nahezu perfekte Geschlossenheit bei Abstimmungen die Norm ist. Durchschnittlich erreichen die Landtagsfraktionen einen Wert von 0,95 beim Index of Agreement bzw. 97,6, wenn man den Rice-Index zu Grunde legt. Selbst vereinzelte deutliche Abweichungen von dieser Norm rütteln nicht am Funktionieren der parlamentarischen Regierungssysteme in den Ländern. Regierungsfraktionen erreichen praktisch immer hinreichende Geschlossenheit, um Abstimmungen zu gewinnen.

Darüber hinaus können verschiedene Befunde über wichtige Einflussfaktoren auf die Fraktionsgeschlossenheit festgehalten werden. Die weithin einflussreiche und teils empirisch auch bestätigte Erwartung, dass kandidatenzentrierte Wahlsysteme Dissens befördern (Depauw u. Martin 2008, S. 111; dagegen: Sieberer 
2006b; Carey 2007), kann für die deutschen Länderparlamente zumindest auf Fraktionsebene nicht bestätigt werden. Dabei ist zum einen zu bedenken, dass die deutschen Länder für einen Test dieser Systemvariable nicht optimal sind, da für partei- und kandidatenzentrierte Wahlsysteme nur relativ wenige Fälle zur Verfügung stehen. Bessere Einsichten in die Effekte der personal vote dürften Tests auf individueller Ebene bzw. eine feinere Binnendifferenzierung der Kategorie gemischter Wahlsysteme erbringen. Zum anderen kann dieser Befund auch dafür sprechen, dass in Systemen mit sehr starken Parteien Personal-Vote-Anreize eben nicht bis auf das Abstimmungsverhalten wirken, sondern sich nur bei ,weicheren ${ }^{\text {" }}$ Indikatoren wie z. B. individuellen Wahlkampfstilen (Zittel u. Gschwend 2007) niederschlagen. Hierüber verspräche eine kombinierte Untersuchung mehrerer Indikatoren weiteren Aufschluss (Crisp 2007).

Als zentrales neues und deutlich robustes Resultat wurde erbracht, dass der Antrag auf namentliche Abstimmung ein extrem einflussreicher Prädiktor für den Zusammenhalt bei den entsprechenden Abstimmungen ist. Der Index of Agreement steigt im Mittel um knapp fünf Punkte für die Fraktion, die den Namensaufruf begehrt. Dies zeigt deutlich, wie das strategische Kalkül beim Beantragen namentlicher Abstimmungen das resultierende Datensample beeinflusst und es ist zwingend erforderlich, dies bei der Interpretation der Geschlossenheitswerte zu berücksichtigen.

Die Regierungsbeteiligung entfaltet in den Landtagen einen klaren positiven Einfluss auf die Fraktionsgeschlossenheit. Dieser Befund ist umso bedeutender, als es sich mutmaßlich vorwiegend um Abstimmungssituationen handelt, in denen die Geschlossenheit der Regierungsfraktion einem echten Test unterzogen wird: Wie gezeigt wurde, gehen 80 Prozent der namentlichen Abstimmungen auf einen Antrag der Opposition zurück - tendenziell in Fällen, bei denen über den Beschlussgegenstand innerhalb der Parlamentsmehrheit keine perfekte Einigkeit herrscht. Anstatt Streit innerhalb der Regierung vorzuführen, erzeugen durch die Opposition beantragte namentliche Abstimmungen offensichtlich eher ein uneinheitliches Verhalten konkurrierender Oppositionsfraktionen.

Die Überlegung, dass wachsende Regierungsmehrheiten den Anreiz zur Geschlossenheit mindern, konnte nur schwach belegt werden. Wächst die ideologische Distanz zwischen Mehrheits- und Minderheitsfraktionen, steigen die Geschlossenheitswerte in geringem Umfang. Der starke und robuste Effekt der Dummy-Variable für die ersten Wahlperioden in Ostdeutschland weist schließlich darauf hin, dass auch die hier nicht näher diskutierten soziologischen Variablen einen wichtigen Beitrag zur Fraktionsgeschlossenheit leisten.

Auch wenn sich die Studie vorrangig für den Effekt einzelner Faktoren auf die Geschlossenheit interessiert und keine vollständige Erklärung des Phänomens anstrebt, bleibt das äußerst geringe R-Quadrat des Regressionsmodells diskussionswürdig. Das Modell enthält praktisch alle prominenten rationalistischen Erklärungsfaktoren, kann aber über 93 Prozent der Varianz in den Geschlossenheitswerten nicht aufhellen. Vor diesem Hintergrund erscheint es lohnenswert, auch situative Faktoren, wie z. B. das Politikfeld einer Abstimmung, in weitere Erklärungsversuche mit einzubeziehen. 
Der vorliegende Beitrag hat die rationalistische Analyse von Fraktionsgeschlossenheit weiterentwickelt, indem bestehende und neue Hypothesen mit besseren Daten in einem denkbar gut kontrollierbaren Umfeld getestet wurden. Es wäre wünschenswert, künftige Untersuchungen des kollektiven Phänomens Fraktionsgeschlossenheit noch konsequenter auf die Ebene zu verlagern, auf der es entsteht: die individuellen Abgeordneten und ihr Abstimmungskalkül.

\section{Literaturverzeichnis}

Andeweg, Rudy B., und Jacques Thomassen. 2011. Pathways to Party Unity: Sanctions, Loyalty, Homogeneity and Division of Labour in the Dutch Parliament. Party Politics. Im Erscheinen. doi:10.1177/1354068810377188.

Bawn, Kathleen, und Michael F. Thies. 2003. A Comparative Theory of Electoral Incentives. Journal of Theoretical Politics 15:5-32.

Bell, Lauren C., und Jason M. Roberts 2005. Keeping Score: Parties, Interest Groups, and Roll-Call Voting in the U.S. House of Representatives. Beitrag vorbereitet für das 2005 Annual Meeting of the American Political Science Association, 1.9.2005, Washington, DC.

Benedetto, Giacomo, und Simon Hix. 2007. The Rejected, the Ejected, and the Dejected: Explaining Government Rebels in the 2001-2005 British House of Commons. Comparative Political Studies 40:755-781. doi:10.1177/0010414006299095.

Best, Heinrich, und Stefan Jahr. 2006. Politik als prekäres Beschäftigungsverhältnis: Mythos und Realität der Sozialfigur des Berufspolitikers im wiedervereinten Deutschland. Zeitschrift für Parlamentsfragen 37:63-79.

Bowler, Shaun, David M. Farrell, und Richard S. Katz. 1999. Party Cohesion, Party Discipline and Parliaments. In Party Discipline and Parliamentary Government, Hrsg. Shaun Bowler, David M. Farrell und Richard S. Katz, 3-21. Columbus: Ohio State University Press.

Braun, Daniela, Nicole Seher, Markus Tausendpfund, und Ansgar Wolsing. 2010. Einstellungen gegenüber Immigranten und die Zustimmung zur Europäischen Integration. Eine Mehrebenenanalyse. MZES Arbeitspapiere Nr. 136. Mannheim: Mannheimer Zentrum für Europäische Sozialforschung.

Bräuninger, Thomas, und Marc Debus. 2008. Der Einfluss von Koalitionsaussagen, programmatischen Standpunkten und der Bundespolitik auf die Regierungsbildung in den deutschen Ländern. Politische Vierteljahresschrift 49:309-338.

Broscheid, Andreas, und Thomas Gschwend. 2005. Zur statistischen Analyse von Vollerhebungen. Politische Vierteljahresschrift 46:16-26.

Cain, Bruce E., John A. Ferejohn, und Morris P. Fiorina. 1987. The Personal Vote. Constituency Service and Electoral Independence. Cambridge: Harvard University Press.

Carey, John M. 2006. Legislative Organization. In The Oxford Handbook of Political Institutions, Hrsg. Rod A. W. Rhodes, Sarah A. Binder und Bert A. Rockman, 431-454. Oxford/New York: Oxford University Press.

Carey, John M. 2007. Competing Principals, Political Institutions, and Party Unity in Legislative Voting. American Journal of Political Science 51:92-107.

Carey, John M., und Matthew S. Shugart. 1995. Incentives to Cultivate a Personal Vote: a Rank Ordering of Electoral Formulas. Electoral Studies 14:417-439. 
Carrubba, Clifford J., Matthew Gabel, Lacey Murrah, Ryan Clough, Elizabeth Montgomery, und Rebecca Schambach. 2006. Off the Record: Unrecorded Legislative Votes, Selection Bias and Roll-Call Vote Analysis. British Journal of Political Science 36:691704. doi:10.1017/S0007123406000366.

Cheibub, Jose Antonio. 2007. Presidentialism, Parliamentarism, and Democracy. New York: Cambridge University Press.

Cheibub, José A., und Fernando Limongi. 2002. Democratic Institutions and Regime Survival: Parliamentary and Presidential Democracies Reconsidered. Annual Review of Political Science 5:151-179.

Clinton, Joshua D. 2007. Lawmaking and Roll Calls. The Journal of Politics 69:457-469. doi:10.1111/j.1468-2508.2007.00543.x.

Cox, Gary W., und Mathew D. Mccubbins. 1993. Legislative Leviathan. Party Government in the House. Berkeley: University of California Press.

Cox, Gary W., und Mathew D. Mccubbins. 2005. Setting the Agenda. Responsible Party Government in the U.S. House of Representatives. Cambridge: Cambridge University Press.

Crisp, Brian F. 2007. Incentives in Mixed-Member Electoral Systems: General Election Laws, Candidate Selection Procedures, and Cameral Rules. Comparative Political Studies 40:1460-1485. doi:10.1177/0010414007301703.

Davidson-Schmich, Louise K. 2003. The Development of Party Discipline in New Parliaments: Eastern German State Legislatures 1990-2000. Journal of Legislative Studies 9:88-101.

Davidson-Schmich, Louise K. 2006. Becoming Party Politicians. Eastern German State Legislators in the Decade Following Democratization. Notre Dame: University of Notre Dame Press.

Depauw, Sam, und Shane Martin. 2008. Legislative Party Discipline and Cohesion in Comparative Perspective. In Intra-party politics and coalition governments, Hrsg. Daniela Giannetti und Kenneth Benoit, 103-120. Milton Park, Abingdon: Routledge.

Desposato, Scott W. 2005. Correcting for Small Group Inflation of Roll-Call Cohesion Scores. British Journal of Political Science 35:731-744.

Diermeier, Daniel, und Timothy J. Feddersen. 1998. Cohesion in Legislatures and the Vote of Confidence Procedure. American Political Science Review 92:627-640.

Dittberner, Jürgen. 2003. Freies Mandat und politische Geschlossenheit. Widerspruch oder Ergänzung zweier Prinzipien des Parlamentarismus? Zeitschrift für Parlamentsfragen 34:550-564.

Döring, Herbert, und Christoph Hönnige. 2006. Vote of Confidence Procedure and Gesetzgebungsnotstand: Two Toothless German Tigers of Governmental Agenda Control. German Politics 15:1-26.

Eilfort, Michael. 2003. Geschlossenheit und gute Figur. Ein Versuch über die Steuerung von Fraktionen. Zeitschrift für Parlamentsfragen 34:565-582.

Flick, Martina, und Adrian Vatter. 2007. Bestimmungsgründe der Parteienvielfalt in den deutschen Bundesländern. Politische Vierteljahresschrift 48:44-65.

Fritzsche, Erik. 2009. Innerfraktionelle Geschlossenheit im Spiegel der Politikwissenschaft. Stand der Forschung und notwendige Weiterführungen. Zeitschrift für Parlamentsfragen 40:661-682. 
Gallagher, Michael, und Michael Marsh. 1988. Introduction. In Candidate Selection in Comparative Perspective. The Secret Garden of Politics, Hrsg. Michael Gallagher und Michael Marsh, 1-19. London: Sage Publications.

Ganghof, Steffen. 2005. Kausale Perspektiven in der vergleichenden Politikwissenschaft: X-zentrierte und Y-zentrierte Forschungsdesigns. In Vergleichen in der Politikwissenschaft, Hrsg. Sabine Kropp und Michael Minkenberg, 76-93. Wiesbaden: VS Verlag für Sozialwissenschaften.

Ganghof, Steffen. 2010. Review Article: Democratic Inclusiveness: A Reinterpretation of Lijphart's Patterns of Democracy. British Journal of Political Science 40:679-692. doi:10.1017/S0007123410000128.

Hazan, Reuven Y. 2006. Does Cohesion Equal Discipline? Towards a Conceptual Delineation. In Cohesion and Discipline in Legislatures, Hrsg. Reuven Y. Hazan, 1-11. New York: Routledge.

Hix, Simon. 2002. Parliamentary Behaviour with Two Principals: Preferences, Parties, and Voting in the European Parliament. American Journal of Political Science 46:688-698.

Hug, Simon. 2006. Selection Effects in Roll Call Votes. CIS Working Paper 15. Zürich: Center for Comparative and International Studies.

Hug, Simon. 2010. Selection Effects in Roll Call Votes. British Journal of Political Science 40:225-235.

Jensen, Torben K., Peter Esaiasson, und Knut Heidar. 2000. Party Cohesion. In: Beyond Westminster and Congress the Nordic experience, Columbus: Ohio State University Press.

Jun, Uwe, Melanie Haas, und Oskar Niedermayer (Hrsg.). 2008. Parteien und Parteiensysteme in den deutschen Ländern. Wiesbaden: VS Verlag für Sozialwissenschaften.

Kailitz, Steffen. 2008. Ein Unterschied wie Tag und Nacht? Fraktionsgeschlossenheit in Parlamentarismus und Präsidentialismus. Zeitschrift für Politikwissenschaft 18:291-324.

Kam, Christopher. 2009. Party Discipline and Parliamentary Government. Cambridge: Cambridge University Press.

Klingemann, Hans-Dieter, und Bernhard Wessels. 2003. The Political Consequences of Germany's Mixed-Member System: Personalization at the Grass Roots? In MixedMember Electoral Systems. The Best of Both Worlds?, Hrsg. Matthew S. Shugart und Martin P. Wattenberg, 280-296. Oxford: Oxford University Press.

Könen, Susanne. 2009. Wo sind die Rebellen hin? Dissentierendes Abstimmungsverhalten in ost- und westdeutschen Landtagen. Wiesbaden: VS Verlag für Sozialwissenschaften.

Krehbiel, Keith. 1999. Paradoxes of Party in Congress. Legislative Studies Quarterly 24:31-64.

Krehbiel, Keith. 2000. Party Discipline and Measures of Partisanship. American Journal of Political Science 44:206-221.

Kropp, Sabine. 2001. Regieren in Koalitionen. Handlungsmuster und Entscheidungsbildung in deutschen Länderregierungen. Wiesbaden: Westdeutscher Verlag.

Lancaster, Thomas D., und William D. Patterson. 1990. Comparative Pork Barrel Politics Perceptions from the West-German-Bundestag. Comparative Political Studies 22:458-477.

Long, J. Scott, und Jeremy Freese. 2006. Regression Models for Categorical dependent variables using Stata. College Station: Stata Press.

Lundell, Krister. 2004. Determinants of candidate selection. The Degree of Centralization in Comparative Perspective. Party Politics 10:25-47. 
Mackenrodt, Christian. 2008. Wie wichtig ist die Person? Zur Bedeutung von Persönlichkeitsfaktoren von Wahlkreisbewerbern bei Bundestagswahlen. Zeitschrift für Parlamentsfragen 39:69-83.

Manow, Philip, und Thomas Zittel. 2008. Einleitung. Repräsentative Demokratie im deutschen Mischwahlssystem. Zeitschrift für Politikwissenschaft 18:141-145.

Martin, Lanny W., und Georg Vanberg. 2004. Policing the Bargain: Coalition Government and Parliamentary Scrutiny. American Journal of Political Science 48:13-27.

Massicotte, Louis. 2003. To Create or to Copy? Electoral Systems in the German Länder. German Politics 12:1-22.

Miller, Bernhard. 2007. Beyond Cabinet. Bringing in the Power Bases in Coalition Committees. Beitrag vorbereitet für den Workshop "Political Power in Parliamentary Executives”, ECPR Joint Sessions of Workshops, 7-12.5.2007, Helsinki.

Mitchell, Paul. 2000. Voters and Their Representatives: Electoral Institutions and Delegation in Parliamentary Democracies. European Journal of Political Research 37:335-351.

Morgenstern, Scott. 2004. Patterns of Legislative Politics: Roll Call Voting in Latin America and the United States. Cambridge: Cambridge University Press.

Müller, Jochen. 2009. The Impact of the Socio-Economic Context on the Länder Parties' Policy Positions. German Politics 18:365-384.

Müller, Markus M. 2004. Das Landtagswahlrecht von Baden-Württemberg. Oder: die Ohnmacht der Parteiendemokratie? Zeitschrift für Parlamentsfragen 35:288-294.

Müller, Wolfgang C., Marcelo Jenny, Barbara Steininger, Martin Dolezal, Wilfried Philipp, und Sabine Preisl-Westphal. 2001. Die österreichischen Abgeordneten. Individuelle Präferenzen und politisches Verhalten. Wien: WUV Universitätsverlag.

Müller, Wolfgang C., und Kaare Strøm. 1999. Policy, Office, or Votes? How Political Parties in Western Europe Make Hard Decisions. Cambridge: Cambridge University Press.

Norris, Pippa. 2004. Electoral engineering. Voting rules and political behavior. Cambridge: Cambridge University Press.

Owens, John E. 2006. Explaining Party Cohesion and Discipline in Democratic Legislatures: Purposiveness and Contexts. In Cohesion and Discipline in Legislatures, Hrsg. Reuven Y. Hazan, 12-40. New York: Routledge.

Ozbudun, Ergun. 1970. Party Cohesion in Western Democracies: A Causal Analysis. Beverly Hills: Sage Publications.

Pappi, Franz Urban, und Nicole Michaela Seher. 2009. Party Election Programmes, Signalling Policies and Salience of Specific Policy Domains: The German Parties from 1990 to 2005. German Politics 18:403-425.

Patzelt, Werner J. 1996. Deutschlands Abgeordnete. Profil eines Berufsstandes, der weit besser ist als sein Ruf. Zeitschrift für Parlamentsfragen 27:462-502.

Patzelt, Werner J. 1998. Wider das Gerede vom ,Fraktionszwang' Funktionslogische Zusammenhänge, populäre Vermutungen und die Sicht der Abgeordneten. Zeitschrift für Parlamentsfragen 29:323-347.

Patzelt, Werner J. 2006. Party Cohesion and Party Discipline in German Parliaments. In Cohesion and Discipline in Legislatures, Hrsg. Reuven Y. Hazan, 102-115. New York: Routledge.

Patzelt, Werner J. 2007. The Constituency Roles of MPs at the Federal and Länder Levels in Germany. Regional and Federal Studies 17:47-70. 
Reutter, Werner. 2005. Vertrauensfrage und Parlamentsauflösung. Anmerkungen zur verfassungspolitischen Praxis und zur Verfassungspraxis in den Ländern. Politische Vierteljabresschrift 46:655-673.

Rice, Stuart A. 1928. Quantitative Methods in Politics. New York: Russell \& Russell.

Riescher, Gisela, und Bernt Gebauer. 2004. Der baden-württembergische Landtag. In Länderparlamentarismus in Deutschland. Geschichte - Struktur - Funktionen, Hrsg. Siegfried Mielke und Werner Reutter, 53-77. Wiesbaden: VS Verlag für Sozialwissenschaften.

Saalfeld, Thomas. 1995a. On Dogs and Whips: Recorded Votes. In Parliaments and Majority Rule in Western Europe, Hrsg. Herbert Döring, 528-565. Frankfurt a. M.: Campus Verlag.

Saalfeld, Thomas. 1995b. Parteisoldaten und Rebellen. Eine Untersuchung zur Geschlossenheit der Fraktionen im Deutschen Bundestag (1949-1990). Opladen: Leske + Budrich.

Saalfeld, Thomas. 2005. Determinanten der Fraktionsdisziplin. Deutschland im internationalen Vergleich. In Mechanismen der Politik strategische Interaktion im deutschen Regierungssystem, Hrsg. Steffen Ganghof und Philip Manow, 35-71. Frankfurt a. M.: Campus Verlag.

Schoen, Harald, und Thorsten Faas. 2005. Reihenfolgeeffekte bei der bayrischen Landtagswahl 2003: Die Ersten werden die Ersten sein. Zeitschrift für Parlamentsfragen 36:100-116.

Schüttemeyer, Suzanne S. 1998. Fraktionen im deutschen Bundestag 1949-1997. Empirische Befunde und theoretische Folgerungen. Opladen: Leske + Budrich.

Schüttemeyer, Suzanne S., und Roland Sturm. 2005. Der Kandidat - das (fast) unbekannte Wesen: Befunde und Überlegungen zur Aufstellung der Bewerber zum Deutschen Bundestag. Zeitschrift für Parlamentsfragen 36:539-553.

Shugart, Matthew S., Melody Ellis Valdini, und Kati Suominen. 2005. Looking for Locals: Voter Information Demands and Personal Vote-Earning Attributes of Legislators under Proportional Representation. American Journal of Political Science 49:437-449.

Sieberer, Ulrich. 2006a. Agenda Setting in the German Bundestag: A weak Government in a Consensus Democracy. German Politics 15:49-72.

Sieberer, Ulrich. 2006b. Party unity in parliamentary democracies: A comparative analysis. Journal of Legislative Studies 12:150-178.

Sieberer, Ulrich. 2007. ,Aber könnte es nicht auch sein, dass...?‘. Die Auswahl unabhängiger Variablen in X-zentrierten und Y-zentrierten Forschungsdesigns. In Forschungsdesign in der Politikwissenschaft. Probleme - Strategien - Anwendungen, Hrsg. Thomas Gschwend und Frank Schimmelfennig, 253-279. Frankfurt/New York: Campus Verlag. Sieberer, Ulrich. 2010. Behavioral consequences of mixed electoral systems: Deviating voting behavior of district and list MPs in the German Bundestag. Electoral Studies 29:484-496.

Snyder, James M., und Tim Groseclose. 2000. Estimating Party Influence in Congressional Roll-Call Voting. American Journal of Political Science 44:187-205.

Stecker, Christian. 2010. Causes of Roll Call Vote Supply. Journal of Legislative Studies $16: 438-459$. 
Stecker, Christian. 2011 (zur Veröffentlichung angenommen). Namentliche Abstimmungen als Währung individueller Verantwortlichkeit? Eine vergleichende Analyse der deutschen Länderparlamente. Zeitschrift für Vergleichende Politikwissenschaft.

Stratmann, Thomas, und Martin Baur. 2002. Plurality Rule, Proportional Representation, and the German Bundestag: How Incentives to Pork-Barrel Differ across Electoral Systems. American Journal of Political Science 46:506-514.

Thiem, Janina. 2006. Explaining Roll Call Vote Request in the European Parliament. MZES Arbeitspapiere Nr. 90. Mannheim: Mannheimer Zentrum für Europäische Sozialforschung.

Zittel, Thomas. 2008. Die elektronische Wählerkommunikation von Abgeordneten aus vergleichender Perspektive - Medienwandel oder Demokratiewandel? Zeitschrift für Politikwissenschaft 18:185-208.

Zittel, Thomas, und Thomas Gschwend. 2007. Individualisierte Wahlkämpfe im Wahlkreis. Eine Analyse am Beispiel des Bundestagswahlkampfes von 2005. Politische Vierteljabresschrift 48:293-321.

\section{Autorenangaben:}

Dipl.-Pol. Christian Stecker,

Universität Potsdam, Lehrstuhl für Vergleichende Politikwissenschaft, August-Bebel-Straße 89, 14482 Potsdam, stecker@uni-potsdam.de 